\title{
Multifrequency Subpulse SAR: Exploiting Chirp Bandwidth for an Increased Coverage
}

\author{
Federica Bordoni $^{\circledR}$, Gerhard Krieger ${ }^{\circledR}$, Fellow, IEEE, and Marwan Younis, Senior Member, IEEE
}

\begin{abstract}
Spatial resolution and swath width are fundamental quality parameters for spaceborne synthetic aperture radar (SAR) products. They have driven the research on new spaceborne SAR system concepts in the last decades, and a new generation of SAR systems is emerging. Main feature of these future systems is the use of multiple digital channels and new SAR processing techniques. A further characteristic is that they can resort to a large radar signal bandwidth. In this letter, a novel SAR operational mode is presented, denoted as multifrequency subpulse (MFSP). The MFSP exploits the available radar signal bandwidth to increase the imaged swath extension, without the emergence of range ambiguities. The proposed approach is explained both theoretically and by a design example, based on the future German X-band SAR system, HRWS. Moreover, the achievable SAR imaging quality is investigated.
\end{abstract}

Index Terms-Digital beamforming (DBF), high-resolution wide-swath (HRWS), multichannel synthetic aperture radar (SAR).

\section{INTRODUCTION}

$\mathbf{S}$ PATIAL resolution and swath extension are key quality parameters of synthetic aperture radar (SAR) images. Their relevance for the end user has driven the evolution of spaceborne SAR systems in the last decades [1]-[7]. Different tradeoffs between these parameters were achieved by different operational modes, such as ScanSAR and spotlight. This allowed delivering images that, if compared with those acquired in the conventional stripmap mode, provide either a wider swath with a degraded azimuth resolution (ScanSAR) or a finer azimuth resolution on the cost of a reduced coverage (spotlight). Nevertheless, these operational modes did not overcome the fundamental design limitation of conventional spaceborne SAR systems, which must compromise between swath extension and azimuth resolution [4]. The possibility to reach simultaneously high resolution and wide coverage required a new concept for the SAR system architecture and image processing: a new generation of SAR systems emerged, characterized by multiple receive/transmit ( $\mathrm{Rx} / \mathrm{Tx})$ channels, and increasing hardware digitization [1]-[7]. For these multichannel SAR systems new operational modes, relying on the

Manuscript received December 15, 2017; revised June 1, 2018 and July 12, 2018; accepted August 15, 2018. This work was supported by Airbus Defense and Space GmbH under Contract F.45984/GO10006374. (Corresponding author: Federica Bordoni.)

The authors are with the Microwaves and Radar Institute, German Aerospace Centre, 82234 Wessling, Germany (e-mail: federica.bordoni@ dlr.de; gerhard.krieger@dlr.de; marwan.younis@dlr.de).

Color versions of one or more of the figures in this letter are available online at http://ieeexplore.ieee.org.

Digital Object Identifier 10.1109/LGRS.2018.2867723 use of special antenna pattern shapes, waveforms, or multiple subpulses, were conceived in order to improve the quotient between swath width and spatial resolution [3], [6], [8].

Among these operational modes, the multisubpulse (MSP) mode, already proposed by Bordoni et al. [8] within a conference paper, should be pointed out here. As ScanSAR, also the MSP mode is conceived for imaging wide scenes, but differs from the former due to the transmission of multiple subpulses within the same pulse repetition interval (PRI). Specifically, each subpulse is used to illuminate a different subswath, so that, not just one but multiple subswaths are imaged within a single burst. As a result, the MSP mode allows for an improved swath extension and/or an improved spatial resolution if compared to a conventional ScanSAR mode.

Main drawback of the MSP is the mutual interference between subpulses, which results in a critical range ambiguity performance [8]. Indeed, even in systems operating with realtime digital beamforming (DBF) on receive according to the scan-on-receive (SCORE) algorithm [1], the MSP mode showed a poor image quality [8]. Origin of the shortcoming is an ineffective separation of the echoes of interest, returned from the different subswaths. In fact, in MSP mode, the subpulses are chirp signals with the same form and common frequency components, and the separation of the corresponding echoes relies mainly on the spatial filtering obtained by the elevation antenna patterns. In order to improve the performance, already in the paper on the MSP mode [8], it was briefly suggested to send subpulses with mutually disjoint bandwidths. This is the core idea of a new SAR operational mode, denoted as multifrequency subpulse (MFSP) and published in a dedicated German patent disclosure in 2014 [9]. The MFSP mode exploits the available radar signal bandwidth in a novel way: no more exclusively to get a better range resolution, as it is traditionally done, but also for an improved SAR image quality in terms of azimuth resolution and/or swath width.

The MFSP mode has a limited impact on the SAR processing (the echoes from each subswath are processed as in a conventional ScanSAR mode [13]). A deeper impact is involved in another, more recently published, approach exploiting the available bandwidth for improving the overall imaging capability [10]. This technique, denoted as F-SCAN and proposed for the future German satellite, High-Resolution WideSwath (HRWS), uses an especially designed array antenna to implement, both in transmission and reception, a frequencydependent elevation beam squint, thereby avoiding the complexity of a DBF in elevation and introducing a continuous 


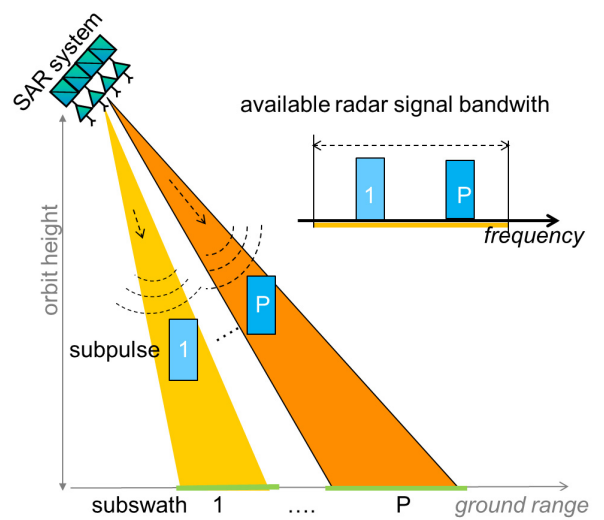

Fig. 1. Transmitted subpulses.

variation versus range of the radio frequency (RF) center frequency used to image the swath. ${ }^{1}$

Indeed, today the idea of a novel exploitation of the available radar signal bandwidth is more topical than ever, since the current technology allows for bandwidth intervals unimaginable until a few years ago. Accordingly, we decide to provide with this letter an extended description and analysis of our MFSP mode.

This letter is organized as follows. The theoretical fundament of the proposed mode is described in Section II. In Section III, based on the actual baseline architecture of the future German multichannel SAR system HRWS, an exemplary system design is presented and the corresponding achievable SAR imaging performance is analyzed. The main results are then outlined in Section IV.

\section{Multifrequency Subpulse Mode}

Main characteristic of the MFSP mode is to use multiple subpulses, occupying disjoint range frequency bands, to image simultaneously multiple subswaths. In detail, P subpulses are sent (simultaneously or sequentially) within the same PRI. Each single subpulse is used to illuminate a different subswath by a dedicated Tx pattern (Fig. 1).

On receive, the echoes from different subswaths are simultaneously collected by $\mathrm{P}$ dedicated patterns. Each of these patterns is designed to optimize the power received from the subswath of interest, and to suppress the range ambiguous signals. Possible Rx patterns include SCORE-based patterns [1], as well as multiple static analog/digital Rx beams. The first option offers the advantage of a high gain and a better range ambiguity suppression with respect to the second option, but involves also a greater hardware complexity. The mutual interference between subpulses is additionally suppressed on receive by means of $\mathrm{P}$ bandpass filters, each one centered over the bandwidth of a different subpulse. The output signals undergo then separately the conventional SAR processing, necessary to image each subswath (Fig. 2).

It is worth remarking that the use of frequency-disjoint bands is crucial for the achievement of a satisfactory image quality and represents the innovative feature of the

\footnotetext{
${ }^{1}$ In MFSP mode, the center frequency varies from subswath to subswath, but remains constant within a subswath.
}

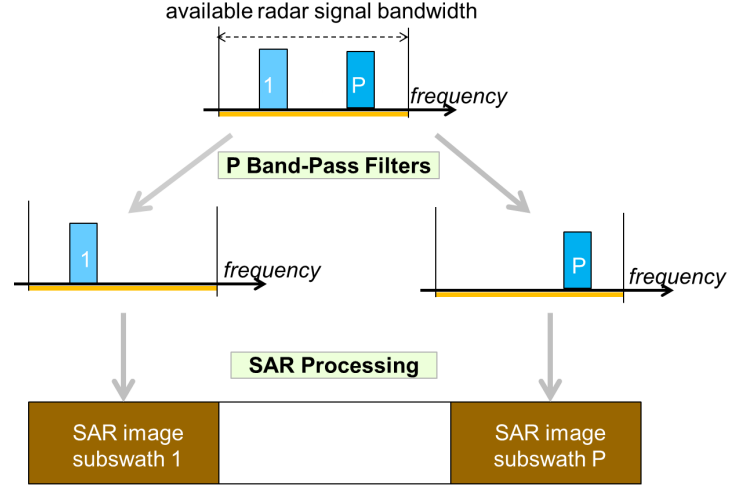

Fig. 2. Separation of the subpulses on receive.

MFSP mode. In fact, it is necessary in order to properly suppress the disturbance generated by the subpulses transmitted in the same PRI. Indeed, if the subpulses would share the same band, three countermeasures could be applied: 1) maximize the interpulse interval; 2) synthetize the elevation pattern on receive according to real-time on-board DBF techniques; and 3) on-ground processing for ambiguity suppression. The first option has a negative impact on the timing constraints, extending the blind regions associated with the Tx event; the other two options generally require a huge antenna height with many digital elements, with a critical impact on the mission costs. In addition, the pattern synthesis could be associated with unwanted gain reduction or even sensitivity to calibration errors and consequently a loss of image quality. Whereas, the on-ground processing requires a huge downlink capability, since the signal received by each antenna element should be sent to the ground.

As explained so far, the use of subpulses with disjoint frequency bands allows the simultaneous imaging of multiple subswaths without mutual interferences. Nevertheless, if the system operates with a single PRF, the overall imaged swath presents gaps, due to the blind regions associated with the Tx event. In order to overcome this limitation, the system operates with different PRFs in burst mode, such as in ScanSAR or TOPS [13]. The extension of the previous description to the burst mode is straightforward and will be further clarified by the example in Section III.

It is worth noting that, since the transmitted subpulses have different bands, the subswath images have different range spectral supports. In the SAR product representing the whole swath, these subimages can be merged seamlessly in a single detected SAR image or delivered within the same SAR complex product without being combined with each other, as current practice for acquisitions in burst mode. The mentioned spectral diversity should not have negative consequence for most SAR applications, since the difference between the center frequencies of the subpulses is small and, in case of interferometric applications, the same subswaths are combined with each other to compute the interferogram ${ }^{2}[14]$.

\footnotetext{
${ }^{2}$ In the interferogram, the slightly different phase-to-path delay relationship causes a phase jump at the edge between subswaths associated with different bands, which can be compensated when deriving the higher level products (digital elevation models, deformation maps, velocity maps).
} 
TABLE I

HRWS SAR SYSTEM REFERENCE PARAMETERS

\begin{tabular}{cc}
\hline \hline Quantity & Value \\
\hline Orbit Height & $514 \mathrm{~km}$ \\
Antenna Tilt Angle & $34.3 \mathrm{deg}$ \\
RF Center Frequency & $9.800 \mathrm{GHz}$ \\
Available Radar Signal Bandwidth & $1200 \mathrm{MHz}$ \\
Tx Peak Power & $7680 \mathrm{~W}$ \\
Feeds Losses (two-way) & $2 \mathrm{~dB}$ \\
Receiver Noise Figure & $3.7 \mathrm{~dB}$ \\
Tx/Rx Antenna (length x height) & $6 \mathrm{~m} \mathrm{x} \mathrm{1.408} \mathrm{m}$ \\
Nr. of Radiators (az. x el.) & $12 \times 64$ \\
Nr. of Digital Rx Az. Chan. & $4 / 2 / 1(\mathrm{SP}), 2 / 1(\mathrm{FP})$ \\
\hline \hline
\end{tabular}

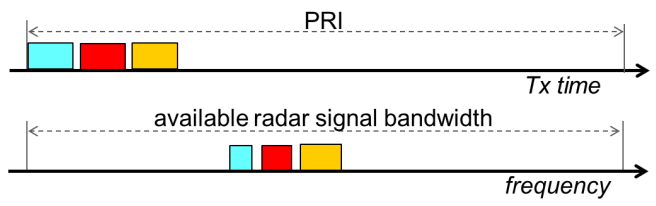

Fig. 3. Tx subpulses represented versus Tx time and frequency.

\section{MFSP APPLIED TO THE HRWS SYSTEM}

In order to exemplify a possible application of the MFSP mode, a system design and a performance analysis are reported. As reference system, the future German satellite, HRWS, is considered. This system is planned as next generation of the TerraSAR-X (TSX) satellite, operating since June 2007.

The main parameters of the HRWS system are reported in Table I. Like TSX, the HRWS is an X-band system orbiting at a height of $514 \mathrm{~km}$. It is based on a planar phased array antenna, $1.4 \mathrm{~m}$ high and $6 \mathrm{~m}$ long. The system is equipped with four digital Rx channels, uniformly distributed along the azimuth direction, which can be used to implement the multichannel reconstruction, according to the MAPS (multiple azimuth phase centers) approach [2], or to provide the system with fully polarimatric capability. In the following, a single polarimetric mode is considered, i.e., all four channels are exploited to implement MAPS.

\section{A. System Design}

The system is assumed to send sequentially three chirp subpulses in each PRI, by using the complete antenna for each single subpulse. The subpulses are separated from each other by an interpulse interval of $3 \mu \mathrm{s}$. The duty cycle of each subpulse is $8 \%$, so that the whole transmission has a duty cycle of $26 \%$. The choice of the duration of the subpulses is a compromise between the necessity to limit the transmission duration and the desire to maximize the average Tx power. In the frequency domain, the subpulses cover disjoint segments of the available radar signal bandwidth (Fig. 3), each with an extension between 90 and $43 \mathrm{MHz}$, so that less than $200 \mathrm{MHz}$ are totally used out of the $1200 \mathrm{MHz}$ available bandwidth.

The system operates in burst mode as ScanSAR [13]: three bursts are used to illuminate in a single pass a swath of $320 \mathrm{~km}$, ranging from about $20^{\circ}$ to $47^{\circ}$ incidence angle. The PRF values are chosen to be around the uniform PRF value $(2535 \mathrm{~Hz})$ [4]. The imaged swath is divided into nine

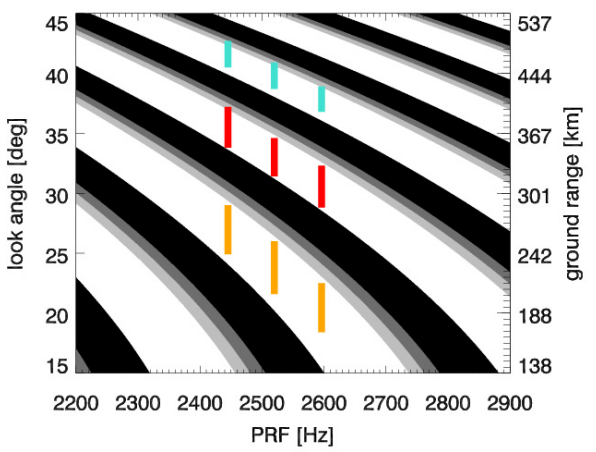

Fig. 4. Timing diagram: the segments denote the imaged subswaths and are colored according to the corresponding subpulse number (see Fig. 3); the dark stripes cover blind regions (the dependence on the subpulse is in gray).

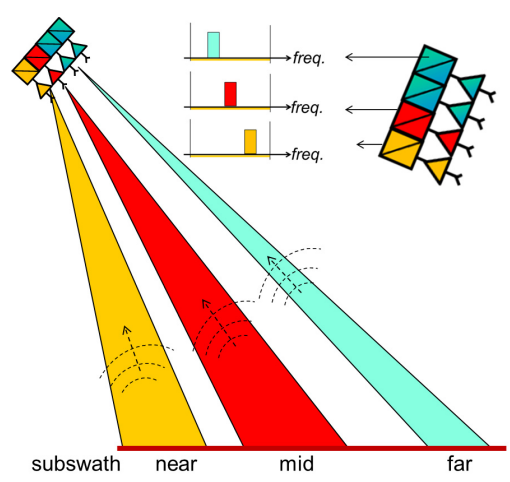

Fig. 5. Subpulse reception.

subswaths: three in near, three in mid, and three in far range, as shown by the timing diagram in Fig. 4 (nadir interference is not considered as a design constraint, see [11]). More in detail, the subswaths in near range are imaged by the third transmitted subpulse, those in mid-range by the second, those in far range by the first. This order of transmission allows optimizing the swath extension w.r.t. the blind regions.

On transmission, the elevation pattern must be switched from subpulse to subpulse, in order to properly illuminate each subswath. In fact, as shown in Fig. 4, each subswath is characterized by a specific elevation angle interval and extension. More in detail, the subswath extension varies between about $4^{\circ}$ and $2^{\circ}$, i.e., about 4 and 2 times the half power beamwidth of the elevation pattern obtained by a uniform excitation of the antenna. Therefore, the Tx elevation beams are widened by a phase-only excitation according to the phase spoiling technique [12].

On receive, as shown in Fig. 5, the subpulse echoes are recorded simultaneously by using the whole antenna. In order to adapt the Rx elevation pattern to the subswath extension, different portions of the antenna are dedicated to different imaged subswaths: 1/4 (16 elements in elevation) of the antenna is used to realize a $\mathrm{Rx}$ elevation pattern pointing toward the near range subswath; $1 / 4$ (16 elements in elevation) of the antenna toward the mid-range subswath; 1/2 (32 elements) of the antenna toward the far range subswath. No taper is assumed for the Rx patterns. The signal received by each portion of the Rx antenna should then be filtered by a bandpass filter over the corresponding subpulse bandwidth. This allows removing the mutual interference between the 


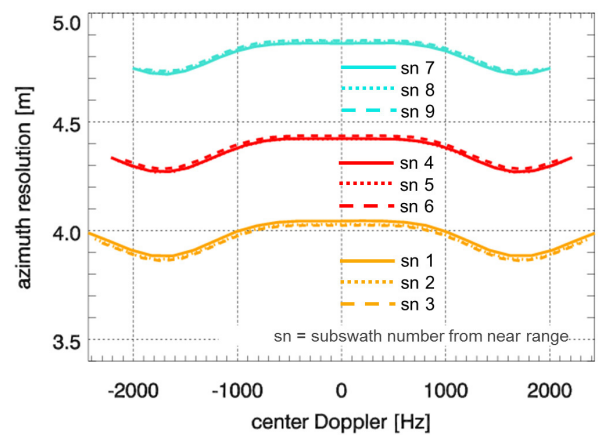

Fig. 6. Azimuth resolution versus Doppler frequency.

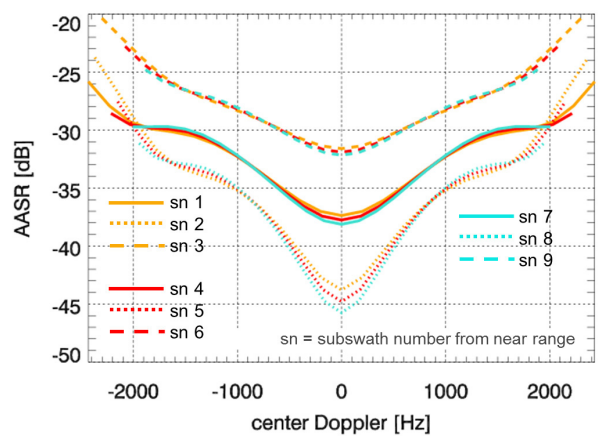

Fig. 7. AASR versus Doppler frequency.

subpulses and also suppressing the range ambiguities. The output of each filter represents the useful signal, backscattered from the corresponding subswath. This can finally undergo the general SAR processing, necessary to image the subswath. It is worth noting that signals received by the three portions of the antenna should not be combined without previously being filtered. Accordingly, an on-board filtering allows reducing the downlink data volume.

As regards the azimuth pattern, on Tx a phase-only taper is employed in order to widen the beam by approximately a factor of 3 (an optimal azimuth resolution would require a broadening by a factor of 4 , but for the present system architecture this would involve an inacceptable performance degradation, due to the reduction of gain and the increase of azimuth ambiguities).

\section{B. SAR Imaging Performance}

As mentioned in Section III-A, the swath extension is of about $320 \mathrm{~km}$. The ground range resolution remains below $5 \mathrm{~m}$ over the whole swath. In fact, the chirp bandwidth, used to image the subswaths, varies between about 90 and $43 \mathrm{MHz}$ from near to far range. The azimuth resolution is shown in Fig. 6. Recognizable are the variations over the imaged swath inherent to the ScanSAR mode [13]. Moreover, since the azimuth illumination time is the same for the multiple subswaths illuminated with the same PRF, it varies versus range: it is about $4 \mathrm{~m}$ for the subswaths in near range and degrades to about $4.9 \mathrm{~m}$ for those in far range (the corresponding Doppler bandwidth is about 1580 and $1300 \mathrm{~Hz}$, respectively).

The azimuth ambiguity performance depends mainly on the PRF, but is also affected by the mentioned choice of the illumination time [13]. In this case, the performance improves for the far range subwaths. This can be seen in Fig. 7 showing

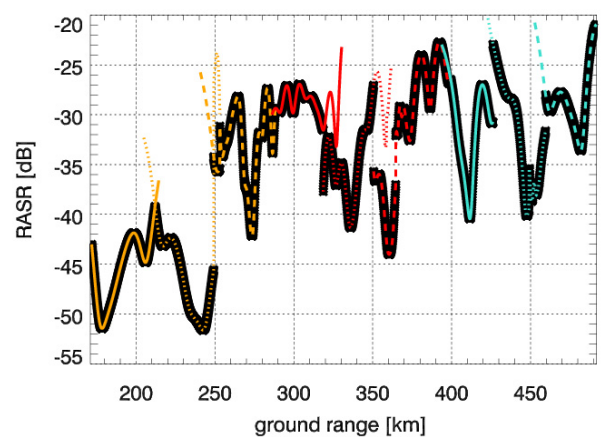

Fig. 8. RASR versus ground range.

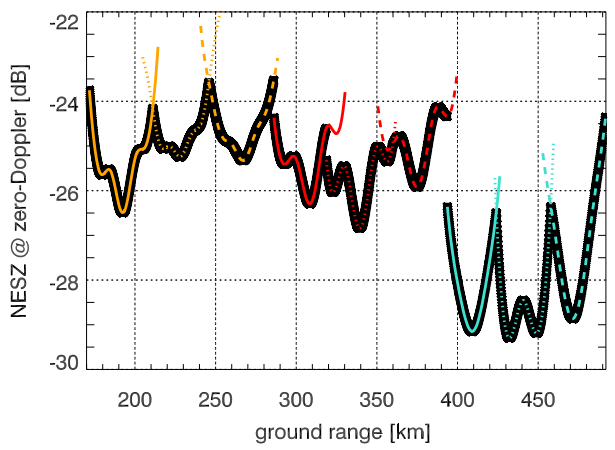

Fig. 9. NESZ at zero-Doppler versus ground range.

the azimuth ambiguity-to-signal ratio (AASR) versus Doppler frequency. The AASR remains below about $-20 \mathrm{~dB}$ for all imaged swaths. More precisely, for the subswaths in near, mid and far range the AASR is below about $-20,-23$, and $-25 \mathrm{~dB}$, respectively.

The range ASR (RASR) versus ground range is shown in Fig. 8. It is computed assuming that the mutual interference between subpulses is suppressed. It reaches the worst value, $-20.5 \mathrm{~dB}$, in far range, whereas in mid and near range remains below about -22.5 and $-27 \mathrm{~dB}$, respectively.

According to the previous results, the total ASR disturbance is below $-20 \mathrm{~dB}$ in the far range subswaths, but reaches a worst value of $-19.2 \mathrm{~dB}$ in near range. Nevertheless, a total ASR below $-20 \mathrm{~dB}$ over the whole access range could be easily obtained by reducing the Doppler processed bandwidth in near/mid-range in order to have $5 \mathrm{~m}$ azimuth resolution for all the subswaths.

Finally, Fig. 9 shows the noise equivalent sigma zero (NESZ) computed at zero-Doppler versus ground range. It is clearly modulated by the phase-spoiled pattern shape and shows an improvement for the far range subswaths due to the higher gain of the Rx pattern. The NESZ at zero-Doppler remains below $-23.5 \mathrm{~dB}$ over the whole imaged swath. The power variation due to the ScanSAR operation is shown in Fig. 10 versus the Doppler frequency: a maximum reduction of about $3.2 \mathrm{~dB}$ is expected. Consequently, the NESZ remains below $-20 \mathrm{~dB}$ over the complete access range. It is worth to note that the scalloping could be mitigated by operating the burst acquisition according to the TOPS technique, instead of ScanSAR [13].

The present analysis demonstrates the possibility to image a swath of about $320 \mathrm{~km}$ with a range and azimuth resolution 


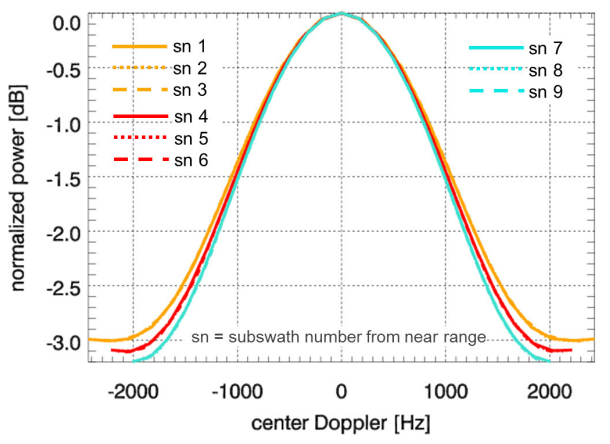

Fig. 10. Power variation versus Doppler frequency.

below $5 \mathrm{~m}$, while keeping the ambiguity disturbance, as well as the NESZ below $-20 \mathrm{~dB}$. In order to appreciate this performance, it is useful to recall that the currently operating TSX system is able to reach a coverage of $100 \mathrm{~km}$ with a resolution in the order of $16 \mathrm{~m}$. The MFSP is therefore suitable to increase the number of acquired independent SAR image pixels by an order of magnitude. It is also remarkable that the mentioned SAR imaging performance is achieved without any real-time DBF on receive in elevation, making the MFSP concept appealing also for very simple systems.

The improvement in terms of imaging performance offered by the MFSP can be explained by comparing the MFSP with a conventional ScanSAR mode. Specifically, the ratio between swath extension and azimuth resolution in ScanSAR mode, would be comparable with that obtained by the MFSP operating with a single subpulse. For instance, in the reported case, if both modes are operating with three bursts at the same azimuth resolution, the MFSP allows imaging six extra subswaths. This improvement is paid first in terms of a larger radar signal bandwidth or alternatively of a loss of range resolution. Moreover, the radiometric and the timing constraints in the two cases are different. In fact, in case of the MFSP the transmitted power should be distributed over a larger surface. In the reported case, $1 / 3$ of the average transmitted power is used to illuminate a single subswath. As a consequence, in order to increase the average Tx power, it could be necessary to extend the subpulse duration.

It is worth emphasizing that the MFSP concept does not rely on the availability of a very wide signal bandwidth, but can be applied also in cases where only a moderate bandwidth is available. This means that it can find useful employment for those SAR imaging applications requiring very wide swaths and medium to low range resolution, such as maritime surveillance, oil spill detection, or mapping of flooding regions.

\section{CONCLUSION}

A novel SAR operational mode, denoted as MFSP, was presented. The MFSP mode was first described in its functional principle and then further explained by means of an exemplary system design, based on the current baseline of the German satellite HRWS, which is considered as a successor to TSX.
The SAR imaging performance was also investigated, showing the possibility for the HRWS to reach a coverage of $320 \mathrm{~km}$ with a spatial resolution of $5 \mathrm{~m} \times 5 \mathrm{~m}$, without any real-time DBF on receive in elevation. Based on the reported analysis, it is possible to conclude that the MFSP offers a novel, simple way to exploit the available radar signal bandwidth in order to significantly extend the imaged swath width and improve the ratio between spatial resolution and swath extension with high overall SAR image quality.

\section{ACKNOWLEDGMENT}

The authors would like to thank their colleagues F. Queiroz de Almeida, P. Prats, M. Rodriguez, R. Scheiber, U. Steinbrecher, and N. Yague for the useful discussions.

\section{REFERENCES}

[1] M. Suess, B. Grafmueller, and R. Zahn, "A novel high resolution, wide swath SAR system," in Proc. IEEE IGARSS, Sydney, NSW, Australia, vol. 3, Jul. 2001, pp. 1013-1015.

[2] G. Krieger, N. Gebert, and A. Moreira, "Unambiguous SAR signal reconstruction from nonuniform displaced phase center sampling," IEEE Geosci. Remote Sens. Lett., vol. 1, no. 4, pp. 260-264, Oct. 2004.

[3] G. Krieger, N. Gebert, and A. Moreira, "Multidimensional waveform encoding: A new digital beamforming technique for synthetic aperture radar remote sensing," IEEE Trans. Geosci. Remote Sens., vol. 46, no. 1, pp. 31-46, Jan. 2008.

[4] N. Gebert, G. Krieger, and A. Moreira, "Digital beamforming on receive: Techniques and optimization strategies for high-resolution wide-swath SAR imaging," IEEE Trans. Aerosp. Electron. Syst., vol. 45, no. 2, pp. 564-592, Apr. 2009.

[5] G. Adamiuk, C. Schaefer, C. Fischer, and C. Heer, "SAR architectures based on DBF for C- and X-band applications," in Proc. EUSAR, Jun. 2014, pp. 1-4.

[6] G. Krieger et al., "SIMO and MIMO system architectures and modes for high-resolution ultra-wide-swath SAR imaging," in Proc. EUSAR, Hamburg, Germany, Jun. 2016, pp. 187-192.

[7] S. Huber et al., "Tandem-L: Design concepts for a next-generation spaceborne SAR system," in Proc. EUSAR, Hamburg, Germany, 2016, pp. 1237-1241.

[8] F. Bordoni, M. Younis, and G. Krieger, "Performance investigation on the high-resolution wide-swath SAR system operating in multisubpulse mode," in Proc. IEEE IGARSS, Munich, Germany, Jul. 2012, pp. $3568-3571$.

[9] F. Bordoni, M. Younis, and G. Krieger, "Synthetik-aperturradarverfahren," German Patent Application DE102013216461A1, Jun. 26, 2014. [Online]. Available: https://depatisnet.dpma.de/Depatis Net/depatisnet?window $=1 \&$ space $=$ main \&content=einsteiger\&action =einsteiger\&switchToLang=en

[10] C. Roemer, "Introduction to a new wide area SAR mode using the F-SCAN principle," in Proc. IEEE IGARSS, Fort Worth, TX, USA, Jul. 2017, pp. 3844-3847.

[11] M. Villano, G. Krieger, and A. Moreira, "Nadir echo removal in synthetic aperture radar via waveform diversity and dual-focus postprocessing," IEEE Geosci. Remote Sens. Lett., vol. 15, no. 5, pp. 719-723, May 2018.

[12] F. Bordoni, M. Younis, N. Gebert, G. Krieger, and C. Fischer, "Performance investigation on the high-resolution wide-swath SAR system with monostatic architecture," in Proc. IGARSS, Vancouver, BC, Canada, Jun. 2010, pp. 1-4.

[13] N. Gebert, G. Krieger, and A. Moreira, "Multichannel azimuth processing in ScanSAR and TOPS mode operation," IEEE Trans. Geosci. Remote Sens., vol. 48, no. 7, pp. 2994-3008, Jul. 2010.

[14] N. Yague-Martinez et al., "Interferometric processing of Sentinel-1 TOPS data," IEEE Trans. Geosci. Remote Sens., vol. 54, no. 4, pp. 2220-2234, Apr. 2016. 